\title{
PRIMARY LYMPHOMA OF THE PROSTATE: A RARE CAUSE OF URINARY OBSTRUCTION
}

\author{
ALBERTO A. ANTUNES, MARCOS DALL'OGLIO, MIGUEL SROUGI \\ Discipline of Urology, Paulista School of Medicine, Federal University of São Paulo (UNIFESP), \\ São Paulo, Brazil
}

\begin{abstract}
Primary lymphoma of the prostate is rare. It represents $0.09 \%$ of prostate neoplasias. The authors report the case of a 42-year old patient presenting urinary obstruction and renal failure due to primary lymphoma of the prostate.

We discuss the clinical manifestations of this disease, emphasizing that systemic chemotherapy represents the initial and preferential therapeutic method
\end{abstract}

Key words: prostatic neoplasms; lymphoma; ureteral obstruction

Int Braz J Urol. 2004; 30: 410-2

\section{INTRODUCTION}

Primary lymphoma of the prostate is rare. It represents $0.09 \%$ of prostate neoplasias and $0.1 \%$ of all non-Hogdkin lymphomas (1). Secondary involvement of the gland is the most common presentation in such cases (2).

The authors report the case of a patient presenting infravesical obstruction and renal failure resulting from prostate lymphoma, and discuss the main clinical features of this rare neoplasia.

\section{CASE REPORT}

A 42-year old man was seen at our service, complaining of weak urinary stream and dysuria for 10 days. Digital rectal examination showed a voluminous prostate with fibroelastic consistency. Blood testes revealed PSA of $0.7 \mathrm{ng} / \mathrm{mL}$ and serum creatinine of $3.6 \mathrm{mg} / \mathrm{dL}$.

An ultrasonography of the urinary tract showed a 286-gram prostate with moderate bilateral uretero-hydronephrosis. Nuclear resonance imaging was normal in thorax, but the abdominal and pelvic scan evidenced a voluminous expansive and infiltrative lesion exclusive of the prostate, with intense impregnation, measuring $10 \times 8 \mathrm{~cm}$ (Figure-1), bilateral uretero-hydronephrosis, and absence of lymph nodal involvement. Bone scintigraphy was negative for secondary lesions. Patient evolved to anuria, and subsequently underwent bilateral nephrostomy.

Prostate biopsy was performed, identifying a diffuse large cell lymphoma, whose immunohistochemical test was positive for antibodies CD45 (UCHL1-DAKO) and CD20 (L26-DAKO), characterizing B-cell lymphoma (Figure-2). Using the King \& Cox modified criteria, the diagnosis of primary extranodal lymphoma of the prostate was confirmed (stage IAE) (1).

Patient initiated cytotoxic chemotherapy with cyclophosphamide, doxorubicin, vincristine and etoposide. After the second cycle, he showed significant clinical and laboratorial improvement, with spontaneous voiding and creatinine levels of $1.5 \mathrm{mg} /$ dL. 


\section{COMMENTS}

Approximately one third of non-Hodgkin lymphomas occur in extranodal sites. Primary lymphomas of the prostate represent $0.1 \%$ of cases, and many times, it is incidentally found following prostate surgery for resolving bladder obstruction (1).

Primary lymphomas of the prostate occur in men aged 60 years in average. The most frequent presentation forms are obstructive urinary symptoms, and obstruction can lead to renal failure (1-3). The occurrence of systemic symptoms such as fever or weight loss, identification of hepatosplenomegaly and inguinal lymphadenopathy on physical examination, or changes in laboratory tests are uncommon. The digital rectal examination can reveal an extremely enlarged prostate with normal consistency, with unaltered PSA (2).

The definition of primary lymphoma of the prostate is based in several criteria. The main symptoms are urinary, the disease occurs predominantly in the prostate, with or without extension to adjacent tissues and there is no involvement of lymph nodes, liver, spleen or blood up to 1 month after diagnosis (3).

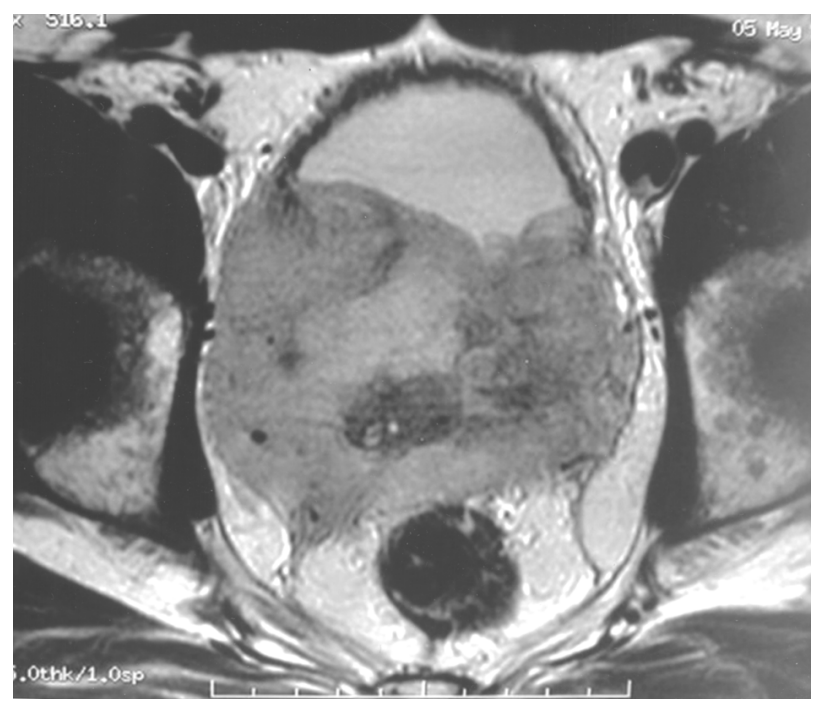

Figure 1 - Magnetic resonance imaging of pelvis, showing a voluminous expansive and infiltrative lesion in prostate.

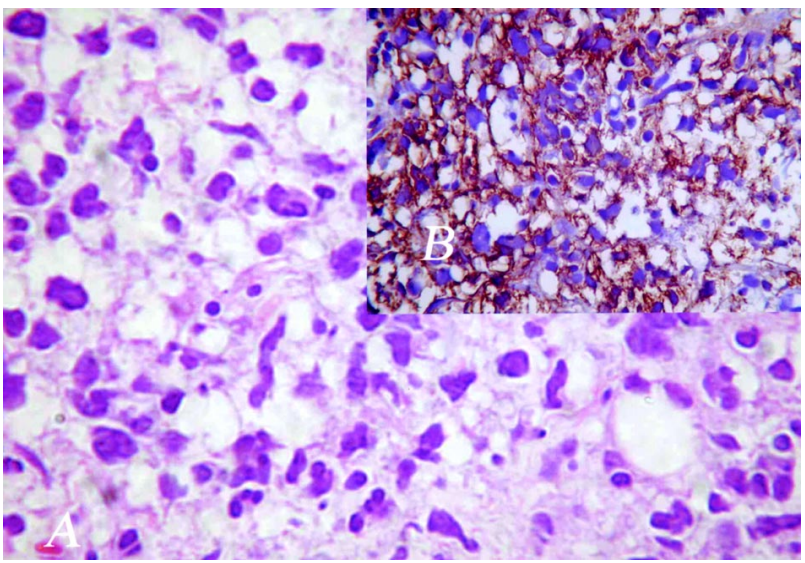

Figure 2-A) - Diffuse large cells lymphoma. B) - Positivity of tumor cells to CD20, characterizing B-cell lymphoma.

Several therapeutic modalities have been reported, including prostatectomy, radiotherapy, chemotherapy, and even cystoprostatectomy $(1,2)$. However, the best therapeutic approach for primary lymphoma of the prostate is chemotherapy based on doxorubicin (3). Among the 23 cases of primary lymphoma of the prostate reported on the Japanese literature, 3 of 5 cases treated with radiotherapy or radical prostatectomy resulted in death or progression of disease. On the other hand, 11 out of 16 cases $(69 \%)$ that received chemotherapy alone or associated with other treatments had a full response (3).

The authors conclude that prostate lymphomas, despite being rare, must be included in differential diagnostic of cases with obstruction of the lower urinary tract, especially in patients with exaggerated enlargement of the gland and previous history of lymphoma in other sites. Chemotherapy constitutes the primary therapeutic approach.

\section{REFERENCES}

1. Sarris A, Dimopoulos M, Pugh W, Cabanillas F: Primary lymphoma of the prostate: good outcome with doxorubicin-based combination chemotherapy. J Urol. 1995; 153: 1852-4.

2. Bostwick DG, Mann RB: Malignant lymphomas involving the prostate. A study of 13 cases. Cancer. 1985; 56: 2932-8. 
3. Fukutani K, Koyama Y, Fujimori M, Ishida T: Primary malignant lymphoma of the prostate: report of a case achieving complete response to combination chemotherapy and review of 22 Japanese cases. Nippon Hinyokika Gakkai Zasshi. 2003; 94: 621-5.

Received: July 20, 2004

Accepted after revision: August 12, 2004

\section{Correspondence address:}

Dr. Alberto Azoubel Antunes

Rua Três de Maio, 17 / 31

São Paulo, SP, 04044-020, Brazil

E-mail: betoazoubel@yahoo.com.br 